\title{
Color stability of self-adhering composite resins in different solutions
}

\section{Trwałość barwy samoadhezyjnych żywic kompozytowych w różnych roztworach}

\author{
Sara Valizadeh ${ }^{1,2, A, D-F}$, Zohreh Asiaie ${ }^{2, B, D, F}$, Nazanin Kiomarsi ${ }^{2, A, E, F}$, Mohammad Javad Kharazifard ${ }^{3, B, C, F}$ \\ ${ }^{1}$ Laser Research Center of Dentistry, Dentistry Research Institute, Tehran University of Medical Sciences, Iran \\ ${ }^{2}$ Department of Restorative Dentistry, School of Dentistry, Tehran University of Medical Sciences, Iran \\ ${ }^{3}$ Dental Research Center, Dentistry Research Institute, Tehran University of Medical Sciences, Iran \\ A - research concept and design; $\mathrm{B}$ - collection and/or assembly of data; $\mathrm{C}$ - data analysis and interpretation; \\ $D$ - writing the article; $E$ - critical revision of the article; $F$ - final approval of the article
}

Address for correspondence

Sara Valizadeh

E-mail: valizadeh_s@sina.tums.ac.ir

Funding sources

None declared

Conflict of interest

None declared

Acknowledgements

This article was part of an MD thesis, supported by Tehran University of Medical Sciences in Iran. (grant No. 9023102006).

Received on July 12, 2019

Reviewed on September 30, 2019

Accepted on November 8, 2019

Published online on March 31, 2020

Cite as

Valizadeh S, Asiaie Z, Kiomarsi N, Kharazifard MJ. Color stability of self-adhering composite resins in different solutions. Dent Med Probl. 2020;57(1):31-38. doi:10.17219/dmp/114099

D0I

10.17219/dmp/114099

Copyright

๑ 2020 by Wroclaw Medical University

This is an article distributed under the terms of the

Creative Commons Attribution 3.0 Unported License (CC BY 3.0)

(https://creativecommons.org/licenses/by/3.0/)

\begin{abstract}
Background. The success of composite resin restorations depends to a great extent on their color stability. However, discoloration is still a problem in composite resin restorations.

Objectives. The aim of the study was to evaluate the effect of different staining solutions on the color stability of composite resins.

Material and methods. A total of 96 composite disks, $2 \mathrm{~mm}$ in height and $8 \mathrm{~mm}$ in diameter, were fabricated of 3 commercially available composite resins. The samples were divided into 4 groups of 8 and were immersed in 4 staining solutions: coffee, tea, soda, and artificial saliva. The color parameters of the samples were measured and recorded before as well as 2, 4 and 8 weeks after immersion by spectrophotometry, using the CIELAB color space. A color change $(\Delta E) \leq 3.3$ was considered the acceptable threshold for visual perception. The results were analyzed using the one-way analysis of variance (ANOVA) and Tukey's post hoc test $(p<0.05)$.

Results. All the composite resins in the study showed discoloration in all the staining solutions. The $\triangle E$ of Vertise ${ }^{\text {TM }}$ Flow was the highest in the tea solution. The lowest $\Delta E$ occurred in the Filtek ${ }^{T M}$ Z250 composite in artificial saliva.
\end{abstract}

Conclusions. This in vitro study showed that the color stability of tooth-colored restorations can be influenced by dietary habits.

Key words: composite resins, staining, self-adhesive, VITA Easyshade

Słowa kluczowe: żywice kompozytowe, barwienie, samoadhezyjny, VITA Easyshade 


\section{Introduction}

Tooth-colored restorative materials are widely used in modern dentistry due to their stability and color match with natural teeth. ${ }^{1}$ An increased demand for esthetics has greatly contributed to the popularity of these naturally looking dental materials. ${ }^{2}$

Long-term durability and color stability in the oral environment are common concerns encountered in the use of restorative materials, such as glass ionomers, composite resins and dental ceramics. Glass ionomers are widely used as a restorative material, base and liner. Dental ceramics are also known as esthetic, color-stable and biocompatible dental materials. Composite resins are commonly used esthetic restorative materials in dental clinics. ${ }^{3}$ According to the literature, dental ceramics have the highest color stability in the oral environment, followed by composite resins and glass ionomers. ${ }^{2}$ Composite resins are more popular among tooth-colored restorative materials due to their optimal strength and esthetics, low cost and strong bonding to the tooth structure. $^{4}$

In order to obtain the best results, composite resin restorations should have the same appearance as natural teeth and must be able to retain this natural appearance over time. Composite resins have been used as direct restorative materials by dentists for over 40 years now, and are considered an acceptable alternative to amalgam and gold restorations. This is due to the development of new composite resins with improved properties. ${ }^{2,3}$

The appearance of a dental restoration should be similar to that of natural teeth. This is directly related to color harmony with the adjacent natural teeth and to the color stability of the material. ${ }^{4}$ A thorough understanding of differential colorimetry and color space is required to determine and quantify the color change of restorative materials. Spectrophotometers are currently used to assess the color change $(\Delta E)$ of dental materials by measuring the $L^{*}, a^{*}$ and $b^{*}$ color coordinates in the CIELAB color space. ${ }^{5}$ The $L^{*}$ coordinate indicates brightness, the $\mathrm{a}^{*}$ coordinate represents the red or green component (positive $a^{*}$ indicates redness and negative $a^{*}$ represents greenness), whereas the $b^{*}$ coordinate is representative of yellowness or blueness (positive $b$ represents yellowness and negative $b^{*}$ indicates blueness). For instance, after composite polymerization, a change in the $b^{*}$ coordinate toward the negative indicates that the composite has lost its yellowness after polymerization. The $\Delta E$ value is calculated by assessing a change in the 3 color parameters, ${ }^{6,7}$ but the $L^{*}$ coordinate is the most important factor in the esthetic appearance of restorations, as a change in lightness has the greatest impact on color.

However, composite resins applied in the oral cavity often undergo the color change over time. ${ }^{8}$ The advantages of composite resins include their color match with the adjacent teeth and their availability in a wide range of shades, their mechanical compatibility and optimal strength against masticatory forces. However, previous studies have shown the discoloration of composite resins in the oral environment. ${ }^{9-11}$

The success of composite resin restorations depends to a great extent on their color stability over time. The discoloration of tooth-colored materials may occur due to internal or external factors. Internal factors include changes in the color of the material itself, i.e., in the resin matrix or at the matrix-filler interface. ${ }^{12}$ The color of restorations may change under different physical and chemical conditions, such as changes in temperature and humidity, the staining of resin-based materials due to exposure to solutions such as tea, coffee and other drinks, or color instability may be a result of aging. ${ }^{13}$ Lifestyle habits, such as a high consumption of colored beverages or smoking, can result in the color change in composite resin restorations.

Habib et al. reported that chocolate, milk and orange juice are among the children's drinks that have the greatest impact on the color stability of composite resins. ${ }^{14}$ Malavasi et al. compared the color stability of 2 self-adhesive composites and a nanofill composite, and showed that the Vertise ${ }^{\mathrm{TM}}$ Flow composite had the highest color stability. ${ }^{15}$ Reddy et al. found that coffee caused the greatest discoloration in the 3 types of composite resins studied (nanohybrid, microhybrid and hybrid), whereas nanocomposites showed the least color change. ${ }^{16}$

Despite the fact that various studies have evaluated the color stability of various restorative materials, further studies are needed on newly introduced toothcolored restorative materials. In addition, in order to achieve an acceptable color match, attention has to be paid to the important role of the color stability of restorative materials. Thus, it is imperative to evaluate the color stability of newly released and highly popular products.

Advances in the composition of composite resins have resulted in the recent introduction of self-adhesive composites. Only a few studies are available on various aspects of self-adhesive composites. This study aimed to evaluate the effect of different staining solutions on the color stability of composite resins, including a selfadhesive one.

\section{Material and methods}

This was an in vitro experimental study. Table 1 presents the characteristics of the composite resins used in this study: Vertise Flow, Premise ${ }^{\mathrm{TM}}$ Flow (both from Kerr Corp., Scafati, Italy) and Filtek ${ }^{\mathrm{TM}}$ Z250 (3M, Maplewood, USA). The A2 shade was used, because it is the composite resin shade most widely used in dentistry. 
Table 1. Composite resins used in the study and their composition

\begin{tabular}{|c|c|c|c|}
\hline Composite resin & Composition & Lot No. & Manufacturer \\
\hline Vertise Flow & $\begin{array}{l}\text { Filler: } \\
\text { - } \text { prepolymerized fillers, containing } 0.7 \mu \mathrm{m} \text { barium glass } \\
\text { - } 1 \mu \mathrm{m} \text { barium glass } \\
\text { - } 10-40 \mathrm{~nm} \text { nano-sized colloidal silica } \\
\text { - } 40 \mathrm{~nm} \text { nano-sized ytterbium fluoride } \\
\text { Resin: } \\
\text { - GPDM adhesive monomer; incorporates the Kerr OptiBond }{ }^{\mathrm{TM}} \text { adhesion technology }\end{array}$ & 3427056 & Kerr Corp., Scafati, Italy \\
\hline Premise Flow & $\begin{array}{l}\text { Filler: } \\
\text { - } 75.5 \text { wt\% barium glass, prepolymerized fillers and silica nanoparticles } \\
\text { Resin: } \\
\text { - Bis-GMA, Bis-EMA, TEGMA, light-cure initiators, and stabilizers }\end{array}$ & 5867548 & Kerr Corp., Scafati, Italy \\
\hline Filtek Z250 & $\begin{array}{l}\text { Filler: } \\
\text { - Zr/Si (60 v\%) } \\
\text { Resin: } \\
\text { - Bis-GMA, Bis-EMA, UDMA }\end{array}$ & N755574 & 3M, Maplewood, USA \\
\hline
\end{tabular}

GPDM - glycerol phosphate dimethacrylate; Bis-GMA - bisphenol A diglycidyl methacrylate; Bis-EMA - ethoxylated bisphenol A diglycidyl methacrylate; TEGMA - triethylene glycol dimethacrylate; UDMA - urethane dimethacrylate.

\section{Sample preparation}

Thirty-two samples were prepared of each material using a plexiglass mold, $8 \mathrm{~mm}$ in diameter and $2 \mathrm{~mm}$ in height. The mold was placed on a glass slide and the composite was applied into the mold. Then, another glass slide was placed over the mold and compressed by placing a $5-\mathrm{kg}$ weight on the top of it for $3 \mathrm{~min}$ to ensure the uniformity of the sample thickness and to eliminate voids. The samples were light-cured using the overlapping technique for $40 \mathrm{~s}$ on each side (80 s in total). Lightcuring was performed using the LED.D light-curing unit (Guilin Woodpecker Medical Instrument Co., Ltd., Guilin, China) at an intensity of $800-850 \mathrm{~mW} / \mathrm{cm}^{2}$. The light intensity was checked after every 5 samples by the LM-1 radiometer (Guilin Woodpecker Medical Instrument Co., Ltd.). The tip of the light guide was put in contact with the glass slide during the light-curing process. The distance between the light source and the sample was standardized using a standard slide. Each sample was then attached to a thread and immersed in the respective solution. The samples were then placed in distilled water for $24 \mathrm{~h}$ to ensure complete polymerization. All samples were polished under a gentle stream of water using 1,000-, 1,500and 2,000-grit abrasive papers to obtain a homogeneous polished surface and to eliminate possible contamination. This was done to minimize the color change due to the surface roughness of the samples and to ensure that the measured color change was due to the inherent properties of the composites. To ensure the uniformity of the surfaces of the samples at all stages, finishing and polishing were done by the same technician, with uniform pressure and the same number of movements; the final thickness of the disks after the completion of polymerization and polishing was $2 \mathrm{~mm}$. A caliper was used to ensure a uniform thickness throughout the disks.
The color parameters were then evaluated using the VITA Easyshade ${ }^{\circledR}$ spectrophotometer (Vident, Inc., Brea, USA). Using this device, the samples were studied in the CIELAB color space, and the 3 color parameters of $L^{*}$ (brightness), $a^{*}$ (red-green) and $b^{*}$ (yellow-blue) were recorded as the baseline color parameters.

\section{Immersion in staining solutions and the discoloration process}

The 32 samples were randomly divided into 4 groups for immersion in 4 solutions $(n=8)$. Each sample was completely immersed vertically in the respective solution using a piece of thread attached to it. The vertical position minimized the discoloration of the surface of the sample. The samples were immersed in the solutions in such a way so as to prevent them from touching each other or the wall. Thus, the samples were only in contact with the solution.

\section{Preparing staining solutions}

Coffee: $5 \mathrm{~g}$ of coffee (Farmand Chocolate Co., Tehran, Iran) was added to $250 \mathrm{~mL}$ of water, placed on a low-heat flame and removed before boiling (according to the manufacturer's instructions); the solution was then filtered using a paper filter.

Tea: A $2 \times 2$-inch tea bag (Debsh Tea Co., Tehran, Iran) was immersed in $250 \mathrm{~mL}$ of water of a temperature of $100^{\circ} \mathrm{C}$ for $60 \mathrm{~s}$.

Artificial saliva was prepared in the laboratory of the Dental Research Center of Tehran University of Medical Sciences in Iran, according to the standard procedure.

Soft drink: Coca-Cola ${ }^{\circledR}$ (Khoshgovar Co., Mashhad, Iran). The samples were immersed in the solutions for $3 \mathrm{~h}$ per day for 8 weeks $(168 \mathrm{~h})$. To maximize the simulation of clinical conditions, the coffee, tea and artificial saliva 
solutions were placed in the Abzar Pezeshki incubator (Kavoosh Mega Co., Tehran, Iran) at $37^{\circ} \mathrm{C}$ (oral temperature), and the cola was placed in a refrigerator at $4^{\circ} \mathrm{C}$ for $3 \mathrm{~h}$ per day.

The solutions were prepared fresh daily. The samples were gently cleansed each time using water and a soft toothbrush for $30 \mathrm{~s}$ to remove any debris due to immersion, and were kept in artificial salvia during the intervals between immersions.

The color parameters of the samples were measured at 2, 4 and 8 weeks after the start of the experiment using the VITA Easyshade spectrophotometer. The total $\Delta E$ of the samples was calculated according to the following formula:

$$
\Delta E=\left[\left(\Delta L^{*}\right)^{2}+\left(\Delta a^{*}\right)^{2}+\left(\Delta b^{*}\right)^{2}\right]^{1 / 2}
$$

where:

$L^{*}$ - lightness;

$a^{*}$ - red (+)/green (-) color coordinate;

$b^{*}$ - yellow (+)/blue (-) color coordinate.

\section{Statistical analysis}

The data was analyzed using IBM SPSS Statistics for Windows, v. 21 (IBM Corp., Armonk, USA) Descriptive statistics, including mean and standard deviation $(S D)$ were reported. The $\Delta E$ values following immersion in different beverages were compared using the one-way analysis of variance (ANOVA). Pairwise comparisons were performed using Tukey's test. The level of significance was set at 0.05 .

\section{Results}

The one-way ANOVA showed that the effects of the type of composite and the type of solution on discoloration were significant at all time points $(p<0.001)$, but the interaction effect of these 2 variables (type of composite and type of solution) on $\Delta a^{*}, \Delta b^{*}, \Delta L^{*}$, and $\Delta E$ was not significant at 2 weeks $(p=0.431), 4$ weeks $(p=0.170)$ or 8 weeks $(p=0.362)$.

The results of the study showed that all the composites tested underwent discoloration over time in all solutions, and that staining increased over time.

The lowest mean $\Delta E$ at the end of the study (8 weeks) was noted in Premise Flow following immersion in artificial saliva $(\Delta E=2.25)$, whereas the highest mean $\Delta E$ was recorded for Vertise Flow following immersion in tea $(\Delta E=27.24)$.

Table 2 presents the mean $\Delta E$ of the composite resins after immersion in different solutions for different periods of time.

After 2 weeks of immersion, the two-way ANOVA showed that the interaction effect of the type of composite and the type of solution on color stability was not significant $(p=0.431)$, but the effects of the type of composite and the type of solution were significant $(p<0.001)$. Tukey's test was used for the pairwise comparisons of the composite resins and showed that the difference between Vertise Flow and the 2 other composites was significant $(p<0.001)$. There was no significant difference in the color stability of Filtek Z250 and Premise Flow ( $p=0.949)$. The pairwise comparisons of the solutions showed that there was a significant difference between all solutions $(p<0.001)$, except for cola and artificial saliva $(p=0.799)$.

The highest $\Delta E$ at the end of the $2^{\text {nd }}$ week was related to the Vertise Flow composite following immersion in coffee and the slightest $\Delta E$ was noted in the Filtek Z250 composite following immersion in artificial saliva.

After 4 weeks of immersion, the two-way ANOVA showed that the interaction effect of the type of composite and the type of solution was not significant $(p=0.170)$, but the effects of the type of composite and type of solution on color stability were significant $(p<0.001)$. Tukey's test was used for the pairwise comparisons of the composites and it was found that the difference between Vertise Flow and the other 2 composites was significant $(p<0.001)$. There was no significant difference in this regard between Filtek Z250 and Premise Flow $(p=0.896)$. The results of the pairwise comparisons of the solutions in terms of the discoloration of different composites were similar to those obtained at 2 weeks - there was no significant difference between cola and artificial saliva ( $p=0.991)$, but significant differences were noted between the remaining solutions $(p<0.001)$.

At the end of the $4^{\text {th }}$ week, the Vertise Flow composite in tea showed the highest discoloration and, as at the end of the $2^{\text {nd }}$ week, the Filtek Z250 composite in artificial saliva showed the least $\Delta E$.

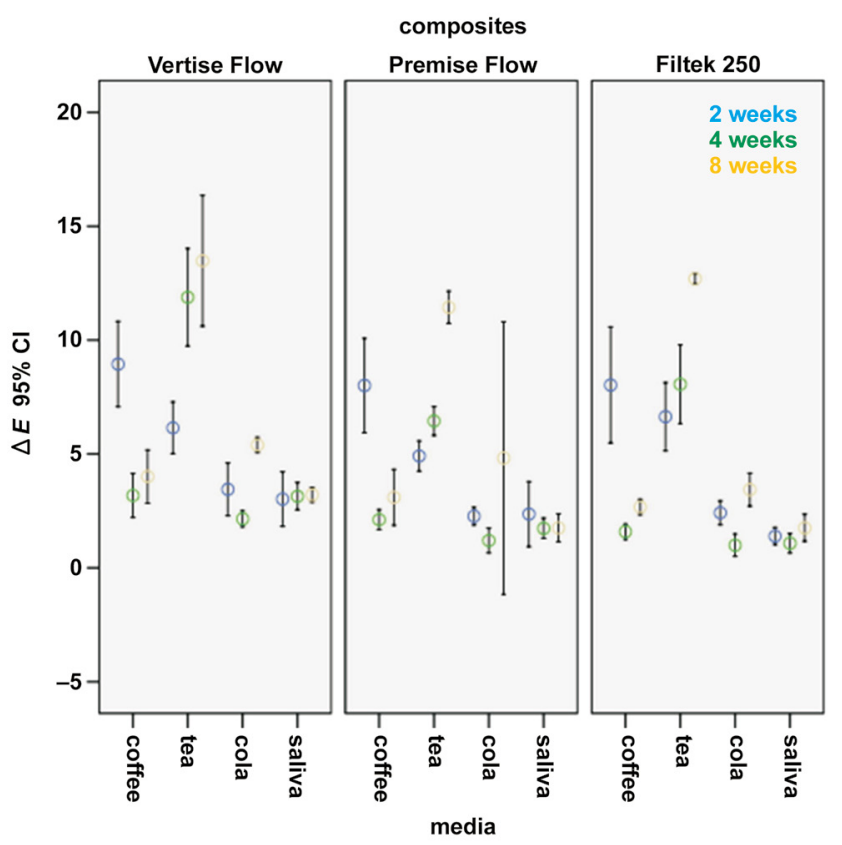

Fig. 1. Composite $\Delta E$ following immersion in the solutions for up to 8 weeks $\mathrm{Cl}$ - confidence interval. 
Table 2. Descriptive information about the color change $(\Delta E)$ values of the composite resins after immersion in different solutions for different periods of time $(n=8)$

\begin{tabular}{|c|c|c|c|c|c|c|}
\hline Medium & Composite & $\begin{array}{c}\Delta E \\
\text { (compared to baseline) }\end{array}$ & Minimum & Maximum & Mean & $S D$ \\
\hline \multirow{9}{*}{ Coffee } & \multirow{3}{*}{ Vertise Flow } & the $2^{\text {nd }}$ week & 6.65 & 13.28 & 8.9456 & 2.23728 \\
\hline & & the $4^{\text {th }}$ week & 6.18 & 18.00 & 11.2526 & 3.66832 \\
\hline & & the $8^{\text {th }}$ week & 9.53 & 21.79 & 14.8293 & 4.53672 \\
\hline & \multirow{3}{*}{ Premise Flow } & the $2^{\text {nd }}$ week & 5.60 & 12.57 & 8.0065 & 2.48011 \\
\hline & & the $4^{\text {th }}$ week & 7.34 & 15.22 & 9.8868 & 2.78348 \\
\hline & & the $8^{\text {th }}$ week & 8.22 & 20.97 & 11.8908 & 4.51950 \\
\hline & \multirow{3}{*}{ Filtek Z250 } & the $2^{\text {nd }}$ week & 5.04 & 15.10 & 8.0277 & 3.04583 \\
\hline & & the $4^{\text {th }}$ week & 6.06 & 16.46 & 9.4160 & 3.12565 \\
\hline & & \multirow{2}{*}{$\begin{array}{l}\text { the } 8^{\text {th }} \text { week } \\
\text { the } 2^{\text {nd }} \text { week }\end{array}$} & 7.34 & 18.57 & 11.0461 & 3.37960 \\
\hline \multirow{9}{*}{ Tea } & \multirow{3}{*}{ Vertise Flow } & & 4.64 & 8.46 & 6.1452 & 1.35659 \\
\hline & & the $4^{\text {th }}$ week & 12.48 & 19.79 & 16.3016 & 2.58172 \\
\hline & & the $8^{\text {th }}$ week & 21.74 & 35.42 & 27.2485 & 5.06403 \\
\hline & \multirow{3}{*}{ Premise Flow } & the $2^{\text {nd }}$ week & 3.67 & 6.18 & 4.9134 & 0.79045 \\
\hline & & the $4^{\text {th }}$ week & 9.17 & 12.62 & 11.1987 & 1.12678 \\
\hline & & the $8^{\text {th }}$ week & 19.92 & 23.85 & 22.2165 & 1.15585 \\
\hline & \multirow{3}{*}{ Filtek Z250 } & the $2^{\text {nd }}$ week & 4.70 & 9.91 & 6.6380 & 1.79172 \\
\hline & & the $4^{\text {th }}$ week & 8.87 & 20.06 & 13.9589 & 3.23060 \\
\hline & & the $8^{\text {th }}$ week & 21.46 & 31.93 & 26.2044 & 3.00274 \\
\hline \multirow{9}{*}{ Coca-Cola } & \multirow{3}{*}{ Vertise Flow } & the $2^{\text {nd }}$ week & 0.90 & 5.39 & 3.4478 & 1.38455 \\
\hline & & the $4^{\text {th }}$ week & 1.56 & 7.60 & 4.5157 & 2.00410 \\
\hline & & the $8^{\text {th }}$ week & 6.00 & 11.96 & 9.0661 & 2.23966 \\
\hline & \multirow{3}{*}{ Premise Flow } & the $2^{\text {nd }}$ week & 1.67 & 2.87 & 2.2686 & 0.46026 \\
\hline & & the $4^{\text {th }}$ week & 1.37 & 3.20 & 2.4581 & 0.57028 \\
\hline & & the $8^{\text {th }}$ week & 2.14 & 24.91 & 5.5623 & 7.83016 \\
\hline & \multirow{3}{*}{ Filtek Z250 } & the $2^{\text {nd }}$ week & 1.60 & 3.36 & 2.4171 & 0.63374 \\
\hline & & the $4^{\text {th }}$ week & 1.45 & 4.38 & 2.5928 & 0.82754 \\
\hline & & the $8^{\text {th }}$ week & 4.10 & 7.66 & 5.1984 & 1.19792 \\
\hline \multirow{9}{*}{$\begin{array}{l}\text { Artificial } \\
\text { saliva }\end{array}$} & \multirow{3}{*}{ Vertise Flow } & the $2^{\text {nd }}$ week & 0.52 & 4.50 & 3.0214 & 1.42778 \\
\hline & & the $4^{\text {th }}$ week & 3.14 & 7.22 & 5.8412 & 1.46400 \\
\hline & & the $8^{\text {th }}$ week & 6.60 & 10.78 & 8.6617 & 1.34524 \\
\hline & \multirow{3}{*}{ Premise Flow } & the $2^{\text {nd }}$ week & 0.74 & 6.00 & 2.3611 & 1.70622 \\
\hline & & the $4^{\text {th }}$ week & 1.24 & 4.73 & 2.3617 & 1.18938 \\
\hline & & the $8^{\text {th }}$ week & 1.34 & 2.92 & 2.2583 & 0.56044 \\
\hline & \multirow{3}{*}{ Filtek Z250 } & the $2^{\text {nd }}$ week & 0.82 & 2.01 & 1.3959 & 0.45073 \\
\hline & & the $4^{\text {th }}$ week & 0.63 & 3.09 & 2.1273 & 0.88576 \\
\hline & & the $8^{\text {th }}$ week & 1.02 & 4.40 & 2.8263 & 1.01785 \\
\hline
\end{tabular}

$S D$ - standard deviation; values in bold indicate the minimum and maximum mean $\triangle E$ in all types of dental composite resins.

After 8 weeks of immersion, the two-way ANOVA revealed that the interaction effect of the type of composite and the type of solution on discoloration was not significant $(p=0.362)$, but the effects of the composite type and the type of solution on the discoloration of the composite resins were significant $(p<0.001)$. Tukey's post hoc test was applied for the pairwise comparisons of the composites and it was found that the difference between Vertise Flow and the other 2 composites was significant $(p<0.001)$, whereas there was no significant difference between Filtek Z250 and Premise Flow $(p=0.926)$. The results of the pairwise comparisons of the solutions in terms of the discoloration of different composites were similar to those obtained at 2 weeks. There was no significant difference between cola and artificial saliva $(p=0.328)$, but significant differences were noted between the remaining solutions $(p<0.001)$. As noted earlier, the greatest $\Delta E$ was recorded for the Vertise Flow composite in tea and the slightest change was seen in the Premise Flow composite in artificial saliva (Fig.1). 


\section{Discussion}

Dental composite resins are widely used these days as esthetic restorative materials. However, the color change in composite restorations is one of the most common reasons for the replacement of these restorations. ${ }^{17,18}$ Color stability is a critical clinical property that affects the esthetic success and longevity of composite restorations. The color change can be considered as an indicator of aging or damage to restorations. ${ }^{19}$

A change in the color of composite restorations over time is a multifactorial process. The color stability of composite resins depends on the resin matrix, filler dimensions, polymerization depth, and color factor, and on chemical differences in the resin components, such as the purity of monomers and oligomers, the type or concentration of the activator, initiator and inhibitor, and the oxidation of the carbon bonding. ${ }^{20}$ Studies have shown that physicochemical reactions, such as visible light radiation, ultraviolet radiation, temperature, and heat, can cause internal color variations in composites over time. ${ }^{21}$ Composite resin materials are continuously exposed to saliva, foods and drinks. These factors as well as oral hygiene and the smoothness of the surface can all affect the color stability of composite restorations..$^{22}$ The aim of the present study was to assess the color stability of a selfadhesive composite resin after immersion in various solutions (coffee, tea, cola, and artificial saliva) as compared with conventional composites.

Natural saliva has a protective effect. By creating a surface barrier, it prevents the staining of the teeth and dilutes staining solutions. ${ }^{23}$ In the present study, for the maximal simulation of clinical conditions, coffee, tea and artificial saliva were incubated at $37^{\circ} \mathrm{C}$ (oral temperature), and the samples immersed in cola were refrigerated at $4^{\circ} \mathrm{C}$.

Simplifying the procedures involved in applying dental materials is useful to minimize errors and to save time. Recently, self-adhesive composites have been introduced to the market. Due to their acidic monomer composition, they bond to the dental structure without requiring an adhesive system. In this study, Vertise Flow, the first self-adhesive composite introduced to the market, was compared with other conventional composites. Vertise Flow has a glycerol phosphate dimethacrylate (GPDM) adhesive, which acts as a bonding agent. This acid phosphate group is used for etching and chemical bonding to calcium ions in the tooth structure. ${ }^{19}$

Polishing may affect the quality of the composite surface, which means the polishing technique can also induce the color change of composite resins..$^{20}$ In the present study, all samples were polished under the same conditions for the purpose of standardization. This was done to minimize the surface roughness and to ensure that the color change calculated at the end of the study was due to the inherent properties of the composite resins.

Coffee, tea and soft drinks (such as Coca-Cola) are among the commonly consumed drinks that have the potential to cause the staining of restorative materials, ${ }^{21,22}$ and were therefore selected for this study.

The CIELAB color scheme was selected for color evaluation in this study, as it is a standard method for measuring color differences based on human perception. ${ }^{23}$ The assessment of composite discoloration can be done visually or using instruments. Instrumental techniques have the advantage of eliminating the subjective interpretations of the color change. Therefore, spectrophotometers and colorimeters are widely used tools to detect the color change in dental restorative materials. ${ }^{24}$

According to previous studies, the $\Delta E$ values $<1$ are not recognizable by the human eye, which can detect $\Delta E>1 .{ }^{25-27}$ The $\Delta E$ values $1<\Delta E<3.3$ are clinically acceptable, whereas any $\Delta E>3.3$ is not. ${ }^{26,27}$ The results of this study showed that the effects of the type of composite and the type of solution on color stability were significant $(p<0.05)$, but the interaction effect of these 2 factors on $\Delta a^{*}, \Delta b^{*}, \Delta L^{*}$, and $\Delta E$ was not significant $(p>0.05)$. The $\Delta E$ value of a composite resin is especially important for anterior restorations. Composite resins decompose over time due to their polymer nature, which leads to their discoloration. ${ }^{28}$

In the present study, the lowest $\Delta E$ value after 8 weeks of immersion was noted in Filtek Z250 in artificial saliva $(\Delta E=1.02)$, whereas the highest $\Delta E$ was noted in Vertise Flow in tea $(\Delta E=35.42)$. Also, significant differences were noted in the color stability of the composites in coffee and tea in comparison with other solutions $(p<0.001)$, and $\Delta E$ for coffee was significantly higher than that for tea, cola and artificial saliva. At 2 weeks, $\Delta E$ for coffee was significantly higher than $\Delta E$ for the other 3 staining solutions. At 4 and 8 weeks, tea, coffee, cola, and artificial saliva caused discoloration in a descending order, and there were significant differences between the $\Delta E$ values for tea and coffee.

All composites showed discoloration in all staining solutions, which increased over time. Composite discoloration is probably due to the external absorption of stains. Coffee and tea cause yellow stains with different polarization. The release of components of a higher polarity (tea) is greater over time. In the first 2 weeks, coffee caused greater discoloration, but at 4 and 8 weeks, the discoloration caused by tea was greater than that caused by other solutions..$^{29,30}$ The discoloration caused by tea is due to the adsorption of polar colorants into the surface of composite resin materials and can be removed by tooth brushing, whereas the discoloration caused by coffee is due to both absorption and adsorption of polar colorants into the surface of the materials. The adsorption and penetration of colorants into the organic phase of the materials are probably due to the compatibility of the polymer phase with the yellow colorants of coffee. ${ }^{31}$ Cola, despite having the lowest $\mathrm{pH}$ of the staining solutions tested, may possibly cause more degradation, but did not cause as much color change as coffee and tea did, probably due to the lack of yellow colorants in its composition. ${ }^{30,31}$ 
Arregui et al. assessed the color stability and water sorption of the composites immersed in distilled water, coffee, cola, and orange juice, and showed that the highest degree of $\Delta E$ was related to tea and coffee. ${ }^{21}$ Orange juice caused a moderate $\Delta E$; the lowest degree of $\Delta E$ was caused by distilled water and cola. ${ }^{21}$ In a study by HasaniTabatabaei et al., the cola and saliva solutions did not cause significant staining in the Filtek ${ }^{\mathrm{TM}}$ Supreme, Tetric ${ }^{\circledR}$ EvoCeram, Filtek Z250, or Tetric ${ }^{\circledR}$ Ceram D composites. ${ }^{27}$

Malekipour et al. examined the discoloration of the $3 \mathrm{M}$ Filtek $^{\mathrm{TM}} \mathrm{Z100}$ composite caused by the tea and coffee solutions, and showed that the highest degrees of staining were related to tea and coffee, whereas water caused the least staining after 14 days. ${ }^{28}$ After 1 day of immersion, the least $\Delta E$ was related to coffee as compared to tea, cola, lemonade, and distilled water. ${ }^{28}$

Arregui et al. evaluated the color stability of the composites immersed in water at $60^{\circ} \mathrm{C}$ for 30 days. The highest degrees of $\Delta E$ were noted in self-adhesive Vertise Flow and GF-10, whereas the Premise Flow composite showed the least $\Delta E .^{2}$ These authors also listed a high temperature as a reason for the decomposition of the resin matrix. ${ }^{2}$

Our results showed that at 2 and 4 weeks, the $\Delta E$ of the Vertise Flow composite was higher than that of the Premise Flow and Filtek Z250 composites $(p=0.02)$; however, the difference between Premise Flow and Filtek Z250 was not statistically significant. At 8 weeks, there was no significant difference in color stability between the composites. A higher degree of $\Delta E$ for Vertise Flow may be due to its high water sorption. ${ }^{25}$ Self-adhesive composites exhibit more hydrophilic properties than other composites, due to carboxylic acids or phosphate groups in their composition. ${ }^{29,30}$ The presence of hydroxyl, carboxyl and phosphate groups in monomers confers hydrophilicity, making selfadhesive composites more susceptible to water sorption. ${ }^{31}$

Arregui et al. assessed the color stability and water sorption of the flowable composites immersed in distilled water, coffee, cola, and orange juice, and showed that the lowest degrees of $\Delta E$ were noted in the Filtek ${ }^{\mathrm{TM}}$ Bulk-Fill composite in distilled water, and in Vertise Flow in coffee and orange juice. ${ }^{21}$ At the same time, Vertise Flow showed the highest degree of water sorption. There were significant correlations between water sorption and solubility as well as between water sorption and $\Delta E .^{21}$

The samples immersed in artificial saliva also showed $\Delta E$ over time due to water sorption by the composites as well as the hydrophilic property of the resin matrix. ${ }^{12}$ Water sorption decreases the longevity of composite resins due to the plasticization of the resin and the formation of microcracks, which progress at the matrix-filler interface and lead to the color change. .1,31 $^{2}$

It seems that the degree of staining of a composite depends on the physical properties of the resin matrix, such as its hydrophobicity and hydrophilicity, rather than on the surface properties of the substance and the size of filler particles. It has been observed that hydrophilic materials exhibit a high degree of water sorption, and consequently show a greater color variation than hydrophobic materials. If the composite can absorb water, it can absorb other drinks and stains as well. ${ }^{27}$

Premise Flow is a medium-viscosity nanofill resin with $0.4 \mathrm{~nm}$ filler size and $75.5 \mathrm{wt} \%$ filler content. The resin matrix of this composite is composed of bisphenol A diglycidyl methacrylate (Bis-GMA), ethoxylated bisphenol A diglycidyl methacrylate (Bis-EMA) and triethylene glycol dimethacrylate (TEGMA). Filtek Z250 is a microhybrid composite. It has silica and zirconia fillers, measuring $0.01-0.50 \mu \mathrm{m}$, and containing $78 \mathrm{wt} \%$ and $60 \mathrm{v} \%$ of mineral fillers. The resin matrix of Filek Z250 consists of Bis-GMA, Bis-EMA and urethane dimethacrylate (UDMA).

Ethoxylated bisphenol A diglycidyl methacrylate is a type of ethoxylated Bis-GMA, which is highly hydrophilic and has no reactive hydroxyl group in its main polymer chains; it should therefore exhibit insignificant water sorption. Yap and Wee also showed that Bis-EMA-based composites were very resistant to the adverse effects of foods on color stability. ${ }^{12}$ On the other hand, composites with UDMA or Bis-GMA modified with UDMA were more resistant to the color change and water sorption than those with the Bis-GMA base. ${ }^{32}$

The size and distribution of filler particles are also related to the color change. Composites with larger filler particles are more susceptible to the color change from water storage than those with smaller filler particles, which is due to the hydrolysis at the filler-matrix interface. ${ }^{24}$ Ertaş et al. reported that Filtek Z250 had the lowest degree of staining as compared to nanohybrid composites. ${ }^{23}$

Liebermann et al. evaluated the color stability of selfadhesive composites in different solutions for 1 year, and concluded that self-adhesive composites had high water sorption and a high $\Delta E .^{30}$

In our study, the $\Delta E$ values were $>3.3$ for all 3 composite resins, which was clinically unacceptable. Several factors may be responsible for this finding. First of all, not using a toothbrush with toothpaste during the study could have resulted in a reduction in the oral hygiene status in comparison with conditions in the oral cavity. Saliva has a protective and diluting effect in the oral cavity, and consequently prevents the staining of the teeth and dental restorations. In addition, in this in vitro study, all samples were immersed in the solutions for $3 \mathrm{~h}$ continuously, which is different from the clinical setting. Thus, it may be concluded that the severity of staining which occurs in vitro is higher than that in the clinical setting. ${ }^{31,32}$

Methodological limitations are inherent to all in vitro studies. In this study, the samples had flat surfaces, whereas resin restorations in the oral environment have irregular, convex or concave surfaces. Moreover, in this study, the samples were dipped in static staining solutions, unlike in the oral cavity, where solutions are in a dynamic state. Last but not least, factors such as thermal alterations or abrasion were not simulated in this study. ${ }^{33}$ 


\section{Conclusions}

Our findings demonstrated that the self-adhesive composite resin tested in the study underwent greater discoloration after immersion in the staining solutions than the conventional composites. Coffee after 2 weeks, and tea after 4 and 8 weeks caused a greater color change in all 3 types of composite resins as compared to other solutions.

Since a number of factors play a role in the oral environment, it is difficult to generalize in vitro findings to the clinical behavior of restorative materials in the oral cavity; therefore, in vivo studies are required to obtain more reliable results.

Further studies are needed to clarify the issue, but it can be concluded that the color stability of tooth-colored restorations in the oral environment depends on dietary habits, and can be preserved by limiting the intake of colored foods and drinks.

\section{ORCID iDs}

Sara Valizadeh (10) https://orcid.org/0000-0003-0383-7722

Zohreh Asiaie (D) https://orcid.org/0000-0002-8380-7787

Nazanin Kiomarsi (D) https://orcid.org/0000-0002-6936-5943

Mohammad Javad Kharazifard (D) https://orcid.org/0000-0002-0613-884X

\section{References}

1. de Alencar e Silva Leite ML, Sales da Cunha Medeiros e Silva FD, Saeger Meireles S, Marques Duarte R, Maciel Andrade AK. The effect of drinks on color stability and surface roughness of nanocomposites. Eur J Dent. 2014;8(3):330-336.

2. Arregui M, Giner L, Ferrari M, Mercadé M. Colour stability of selfadhesive flowable composites before and after storage in water. Key Eng Mater. 2015;631:143-150.

3. Dabbagh A, Bindal P, Bindal U, Ramanathan A, Ginjupalli K. Comparative effects of turmeric, coffee, and chewable tobacco on the color stability of tooth-colored restorative materials. Open J Dent Oral Med. 2015;3(3):59-67.

4. Badra VV, Faraoni JJ, Ramos RP, Palma-Dibb RG. Influence of different beverages on the microhardness and surface roughness of resin composites. Oper Dent. 2015;30(2):213-219.

5. Zanetti F, Zhao X, Pan J, Peitsch MC, Hoeng J, Ren Y. Effects of cigarette smoke and tobacco heating aerosol on color stability of dental enamel, dentin, and composite resin restorations. Quintessence Int. 2019;50(2):156-166.

6. Gómez-Polo C, Gómez-Polo M, Celemin-Viñuela A, Martínez Vázquez De Parga JA. Differences between the human eye and the spectrophotometer in the shade matching of tooth colour. J Dent. 2014;42(6):742-745.

7. Karaarslan ES, Bulbul M, Ertas E, Cebe MA, Usumez A. Assessment of changes in color and color parameters of light-cured composite resin after alternative polymerization methods. Eur J Dent. 2013;7(1):110-116.

8. Choi MS, Lee YK, Lim BS, Rhee SH, Yang HC. Changes in surface characteristics of dental resin composites after polishing. J Mater Sci Mater Med. 2005;16(4):347-353.

9. Uchida H, Vaidyanathan J, Viswanadhan T, Vaidyanathan TK. Color stability of dental composites as a function of shade. J Prosthet Dent. 1998;79(4):372-377.

10. Correr GM, Bruschi Alonso RC, Baratto-Filho F, Correr-Sobrinho L, Coelho Sinhoreti MA, Puppin-Rontani RM. In vitro long-term degradation of aesthetic restorative materials in food-simulating media. Acta Odontol Scand. 2012;70(2):101-108.

11. Bagheri R, Burrow MF, Tyas M. Influence of food-simulating solutions and surface finish on susceptibility to staining of aesthetic restorative materials. J Dent. 2005;33(5):389-398.

12. Yap AU, Wee KE. Effects of cyclic temperature changes on water sorption and solubility of composite restoratives. Oper Dent. 2002;27(2):147-153.
13. Villalta P, Lu H, Okte Z, Garcia-Godoy F, Powers JM. Effects of staining and bleaching on color change of dental composite resins. J Prosthet Dent. 2006;95(2):137-142.

14. Habib AN El-Din Ahmed, Abdelmoniem SA, Mahmoud SA. Effect of children's drinks on color stability of different dental composites: An in vitro study. J Clin Pediatr Dent. 2017;41(2):120-125.

15. Malavasi CV, Macedo EM, Souza Kda C, Rego FG, Schneider Jochims LF, Cavalcante LM. Surface texture and optical properties of selfadhering composite materials after toothbrush abrasion. J Contemp Dent Pract. 2015;16(10):775-782.

16. Reddy PS, Sunil Tejaswi KL, Shetty S, Annapoorna BM, Pujari SC, Thippeswamy HM. Effects of commonly consumed beverages on surface roughness and color stability of the nano, microhybrid and hybrid composite resins: An in vitro study. J Contemp Dent Pract. 2013;14(4):718-723.

17. Domingos PAdS, Garcia PPNS, de Oliveira ALBM, Palma-Dibb RG. Composite resin color stability: Influence of light sources and immersion media. J Appl Oral Sci. 2011;19(3):204-211.

18. Rezaei-Soufi L, Shirinzad M. Mirtorabi MS. Comparison of the color stability of silorane-based with methacrylate-based resin composites after accelerated artificial aging [in Persian]. J Mash Dent Sch. 2014;38(3):211-220.

19. Furuse AY, Santana LOC, Rizzante FAP, et al. Delayed light activation improves color stability of dual-cured resin cements. J Prosthodont. 2018;27(5):449-455.

20. Heshmat H, Hajian M, Hoorizad Ganjkar M, Emami Arjomand M. Effect of tea on color change of silorane and methacrylate based composite resins. J Islam Dent Assoc Iran. 2013;25(3):198-202.

21. Arregui $M$, Giner L, Ferrari $M$, Vallés $M$, Mercadé $M$. Six-month color change and water sorption of 9 new-generation flowable composites in 6 staining solutions. Braz Oral Res. 2016;30(1):e123.

22. Gönülol N, Ertaş E, Yılmaz A, Çankaya S. Effect of thermal aging on microleakage of current flowable composite resins. J Dent Sci. 2015;10(4):376-382.

23. Ertaş E, Güler AU, Yücel $A C$, Köprülü H, Güler E. Color stability of resin composites after immersion in different drinks. Dent Mater J. 2006;25(2):371-376.

24. Beltrami R, Ceci M, De Pani G, et al. Effect of different surface finishing/polishing procedures on color stability of esthetic restorative materials: A spectrophotometric evaluation. Eur J Dent. 2018;12(1):49-56.

25. Mahdisiar F, Nasoohi N, Safi M, Sahraee Y, Zavareian S. Evaluating the effect of tea solution on color stability of three dental composite (in vitro) [in Persian]. J Res Dent Sci. 2014;11(1):21-26.

26. Sedrez-Porto JA, Aldrighi Münchow E, Cenci MS, Pereira-Cenci T. Translucency and color stability of resin composite and dental adhesives as modeling liquids - a one-year evaluation. Braz Oral Res. 2017;31:e54.

27. Hasani-Tabatabaei M, Yassini E, Moradian S, Elmamooz N. Color stability of dental composite materials after exposure to staining solutions: A spectrophotometer analysis. J Islam Dent Assoc Iran. 2009;21(1):69-78.

28. Malekipour MR, Sharafi A, Kazemi S, Khazaei S, Shirani F. Comparison of color stability of a composite resin in different color media. Dent Res J (Isfahan). 2012;9(4):441-446.

29. Kaviani A, Ahmadzadeh A, Zarei M. Laboratorial comparison of color stability of resin composites after rebonding with two different adhesive materials. J Dent Med Tehran Univ Med Sci. 2013;26(1):42-47.

30. Liebermann A, Roos M, Stawarczyk B. The effect of different storage media on color stability of self-adhesive composite resin cements for up to one year. Materials (Basel). 2017;10(3):300.

31. Sokolowski G, Szczesio A, Bociong K, et al. Dental resin cements - the influence of water sorption on contraction stress changes and hydroscopic expansion. Materials (Basel). 2018;11(6):973.

32. Nasoohi N, Hoorizad M, Torabzade Tari N. Effect of tea and coffee on color change of two types composite resins: Nanofilled and micro hybrid. J Res Dent Sci. 2011;7(4):18-22.

33. Teo K, Ab-Ghani Z, Ali ZHM. Effects of polishing techniques on the staining of two nano-tooth coloured materials. Sains Malays. 2018;47(4):781-787. 